\title{
Homocysteine and other markers of cardiovascular risk during a manic episode in patients with bipolar disorder
}

Fábria Chiarani, ${ }^{1,2}$ Juliana Fernandes Tramontina, ${ }^{1}$ Keila Maria Ceresér, ${ }^{1,3}$ Maurício Kunz, ${ }^{1,3}$ Leonardo Paim, ${ }^{1}$ Carmen Regla Vargas, ${ }^{2,4}$ Angela Sitta, ${ }^{4}$ Sérgio Pinto Machado, ${ }^{5}$

Angela Terezinha de Souza Wyse, ${ }^{2}$ Flávio Pereira Kapczinski ${ }^{1,2,3,5}$

${ }^{1}$ Molecular Psychiatry Unit and National Science and Technology Institute for Translational Medicine (INCT-TM), Hospital de Clínicas de Porto Alegre (HCPA), Universidade Federal do Rio Grande do Sul (UFRGS), Porto Alegre, RS, Brazil. ${ }^{2}$ Graduate Program in Biological Sciences: Biochemistry, UFRGS, Porto Alegre, RS, Brazil. ${ }^{3}$ Graduate Program in Medical Sciences: Psychiatry, UFRGS, Porto Alegre, RS, Brazil.

${ }^{4}$ Medical Genetics Service, HCPA, Porto Alegre, RS, Brazil. ${ }^{5}$ Department of Psychiatry and Legal Medicine, UFRGS, Porto Alegre, RS, Brazil.

\begin{abstract}
Objective: To evaluate serum levels of different biomarkers associated with cardiovascular disease in patients with bipolar disorder (BD). Patients were prospectively evaluated in two separate instances: during acute mania and after remission of manic symptoms. All measurements were compared with those of healthy controls.

Methods: The study included 30 patients with BD and 30 healthy controls, matched for gender and age. Biochemical parameters evaluated included homocysteine (Hcy), folic acid, vitamin B12, ferritin, creatine kinase (CK) and C-reactive protein (CRP).

Results: Hcy levels were significantly higher in the BD patients, both during mania and after achieving euthymia. When Hcy was adjusted for body mass index, there was no significant difference between patients and controls. Ferritin was the only marker that showed a significant decrease during mania when compared to both euthymic patients and controls. There were no significant differences for folate, vitamin B12, CK and CRP.

Conclusions: These findings do not show an association between alterations of markers of cardiovascular risk during manic episodes. Further studies are necessary to determine factors and mechanisms associated with cardiovascular risk in patients with BD.
\end{abstract}

Keywords: Homocysteine; ferritin; bipolar disorder; cardiovascular disease

\section{Introduction}

Individuals with bipolar disorder (BD) have significantly higher mortality rates than the general population. ${ }^{1}$ Medical diseases are common in patients with BD and may affect the course, severity and treatment of the disorder. ${ }^{2}$

Cardiovascular and cerebrovascular conditions are the leading causes of natural death among patients with $\mathrm{BD}$, after suicide or accidents. ${ }^{1,3}$ It has been hypothesized that patients with a history of mania or hypomania may exhibit a greater prevalence of vascular disease when compared with patients with major depression or other mental disorders. ${ }^{4}$

Hyperhomocysteinemia (HHcy) has been associated with increased risk of cardiovascular and cerebrovascular disease. ${ }^{5} \mathrm{~A}$ meta-analysis of associations between homocysteine (Hcy) levels and cardiovascular disease (CVD) showed that for every $5-\mu \mathrm{mol} / \mathrm{L}$ increase in serum Hcy concentration, the risk of ischemic heart disease increased 20 to $30 \%{ }^{6}$ Studies also indicate that Hcy is

Correspondence: Fábria Chiarani, Laboratório de Psiquiatria Molecular, Hospital de Clínicas de Porto Alegre, Rua Ramiro Barcelos, 2350, CEP 90035-903, Porto Alegre, RS, Brazil.

E-mail: fabriachiarani@yahoo.com.br

Submitted 27 Jan 2012, accepted 14 May 2012. associated with several important components of atherogenesis, including endothelial dysfunction, platelet and immune activation and inflammation. ${ }^{7,8}$

Determinants of HHcy, such as low concentrations of folate and vitamin B12 coenzymes, which are involved in Hcy metabolism, are also associated with increased risk of vascular damage. ${ }^{9,10}$ Whether HHcy itself or low concentrations of vitamin $\mathrm{B} 12$ and folate are the atherogenic factors that trigger vascular disease remains to be determined. Individuals with low folate levels or intake have shown a higher risk of CVD; it is possible that these associations may be independent of Hcy. ${ }^{11}$

Studies show that the iron storage protein ferritin plays an important enzymatic role in folate catabolism. Thus, it may be associated with higher Hcy concentrations by reducing the availability of one-carbon units for remethylation. ${ }^{12}$

Studies have established that depressive symptoms or episodes increase the risk of incident cardiovascular disease. ${ }^{13,14}$ To the best of our knowledge, few studies have examined the relationship between manic episodes and CVD. Results of a prospective cohort study of participants with BD showed that (hypo)manic symptoms were independent predictors of cardiovascular mortality, and that mood disorders may mediate vascular disease. This mediation may occur through mechanisms that are 
independent of and distinct from traditional risk factors. ${ }^{15}$ In this same vein, one study has provided support for the notion that heart rate variability and predictability in BD may indeed be state-dependent. ${ }^{16}$

Creatine kinase $(\mathrm{CK})$ enzymes are essential for energy metabolism. These enzymes connect mitochondrial ATPproducing and cytosolic ATP-consuming processes, and are thus of central importance for cellular energy homeostasis. $^{17}$

C-reactive protein (CRP) is considered a marker of systemic inflammation. Prospective studies have shown a direct association between CRP levels and risk of developing cardiovascular disease. ${ }^{18,19}$

The goal of the present study was to evaluate Hcy, folate, vitamin B12, CRP, ferritin and CK levels within the same patient during manic and euthymic states, and compare these measurements with those of controls.

\section{Methods}

Patients with BD were recruited at the Hospital de Clínicas de Porto Alegre, Porto Alegre, Brazil. All subjects underwent a comprehensive clinical interview by a psychiatrist. The diagnosis of BD was established on the basis of available clinical information. In addition, diagnosis was confirmed with the Structured Clinical Interview for DSM-IV - Axis I (SCID-I) and a standard protocol for assessment of psychopathology and clinical features. Baseline assessment, including blood sampling, was performed at admission to the psychiatric unit. Patients had to fulfill DSM-IV criteria for manic episodes. Treatment was naturalistic and at the discretion of the attending psychiatrist. Patients did not have significant comorbid medical conditions. Mood symptoms were assessed with the Young Mania Rating Scale (YMRS) ${ }^{20}$ and the Hamilton Depression Rating Scale (HAM-D), 21item version. ${ }^{21}$ All raters were trained to administer the scales; the results were assessed for inter-rater reliability. After inclusion and initial evaluation, patients were followed up with weekly screenings until remission of manic symptoms, at which point they were reassessed and blood sampling was carried out again. Patients were considered euthymic if their score was $<7$ on both the YMRS and HAM-D scales.

Controls were required to be free of a personal history of neurological or psychiatric illnesses, and have no firstdegree relatives with a diagnosis of psychiatric disorder. Healthy controls and patients were matched for age and gender. All patients and controls gave written informed consent before entering the study. All procedures described in the study were approved by the local ethics committee.

Five milliliters of blood were withdrawn from each subject by venipuncture and stored in an anticoagulantfree vacuum collection tube. The blood was immediately centrifuged at $3000 \times \mathrm{g}$ for $5 \mathrm{~min}$, and serum was kept frozen at $-80^{\circ} \mathrm{C}$ until assayed.

Hcy levels were estimated by stable isotope dilution and electrospray tandem mass spectrometry, as described by Magera et al. ${ }^{22}$ Hcy was detected through the transition from the precursor to the product ion (m/z 136 to $\mathrm{m} / \mathrm{z} 90)$. Homocysteine- $d_{8}$ was added as internal standard. Serum reference values ranged from 5 to $15 \mu \mathrm{mol} / \mathrm{L}$.

Quantitative folate and vitamin B12 serum levels were measured with a chemiluminescent immunoassay by employing the competitive test principle. Serum ferritin concentrations were determined by chemiluminescent sandwich-type immunoassay.

Measurement of CK levels was carried out using a system in which CK catalyzes the transphosphorylation reaction of ADP to ATP. ATP coupled with hexokinaseG6PD reactions produce NADPH in quantities directly proportional to the quantity of $\mathrm{CK}$ in the sample. The analyses were performed by monitoring the increase in absorbance of NADPH per minute at the wavelength of $340 \mathrm{~nm}$.

Measurement of CRP in serum was performed using high-sensitivity C-reactive protein technique (latex immunoturbidimetric assay). The purified anti-human C-reactive protein antibody, when coated in polystyrene particles and mixed with a sample containing CRP, results in an insoluble complex. This complex results in turbidity, which is proportional to the CRP concentration in the sample.

Biochemical parameters were measured using an ADVIA Centaur immunoassay system (Siemens Healthcare Diagnostics, Tarrytown, NY). Statistical analyses were carried out in the SPSS version 18.0 (SPSS Inc., Chicago, IL, USA); results were considered significant with a p-value $<0.05$ and normality was assessed using the Shapiro-Wilk test.

Comparisons between patients with $\mathrm{BD}$ and between patients and controls were carried out using generalized estimating equations (GEE). The GEE procedure is used for analysis of repeated measures. Cases are assumed to be dependent within subjects and independent between subjects. The Gaussian distribution was evaluated for variables with a symmetric profile, and the gamma distribution for variables with an asymmetric profile. Body mass index (BMI) was included as a covariate in the regression model; results are presented with and without inclusion of BMI in the analysis. Bonferroni correction was applied for multiple comparisons.

\section{Results}

Thirty-nine manic patients were recruited upon admission to a psychiatric inpatient unit. Nine patients were lost during follow-up due to transfer to another medical facility or due to withdrawal of consent to participate. Only the patients who completed follow-up until remission were included in the study. Therefore, 30 patients and 30 healthy controls (15 males and 15 females in each group) were included in the analysis. The mean age of BD patients was $41.15 \pm 15.83$ years, and that of controls, $40.84 \pm 14.95$ years.

The BMI of BD patients and controls, expressed as median and interquartile range, was 27.75 (22.41-31.82) and 26.99 (23.69-29.72) respectively. BMI was calculated as weight divided by the height squared $\left(\mathrm{kg} / \mathrm{m}^{2}\right)$. 
The median time elapsed between onset of the acute episode and remission of symptoms in BD patients was 29.50 [20.50-58.00] days. Median HAM-D and YMRS scores were 3.50 [1.75-5.00] and 27.50 [19.00-30.70] respectively in the manic state and 1.00 [0.00-2.50] and $1.00[0.00-4.00]$ respectively in the euthymic state. Table 1 shows the evaluated parameters.

Of the parameters evaluated, only Hcy levels were higher in BD patients in comparison with controls. The values did not differ within the same BD patient during manic and euthymic states. The difference between patient and control Hcy levels did not persist when BMI was included as a covariate.

No statistically significant differences were found in folate and vitamin B12 levels on comparison between BD patients and controls. These levels persisted in BD patients throughout symptomatic recovery. Serum folate and serum vitamin B12 values were within normal range.

Ferritin levels were within normal range in all subjects. However, these levels were lower in BD patients during mania, both in comparison with BD patients in euthymia and in comparison with healthy controls. This finding persisted when BMI was included as a covariate.

CRP levels of bipolar patients were not significantly different between the manic and euthymic states. No significant differences were observed on comparison between patients and healthy controls. CK levels were originally significantly higher in manic patients; however, the difference occurred due to a few outliers that showed elevated CK levels. The elevation in CK was probably associated with the use of intramuscular medication or with psychomotor agitation. In the present study, we did not collect data on the frequency of use of IM medication or level of physical agitation during follow-up.

\section{Discussion}

$\mathrm{BD}$ is strongly associated with changes in cardiovascular function. The etiology of excess CVD is multifactorial and possibly includes genetic and lifestyle factors, as well as treatment effects. ${ }^{23}$

Moderately increased plasma levels of Hcy and genetic factors associated with enzymatic abnormalities of folic acid and Hcy metabolism play an important role in the development of CVD. ${ }^{24,25}$

Most studies that reported higher Hcy levels in bipolar patients were carried out during euthymia and focused on functional and cognitive deterioration. ${ }^{26-28}$ To our knowledge, this is the first study to assess Hcy levels in the same patients in both states, mania and euthymia; it is also the first study to attempt to correlate Hcy with markers of CVD.

Our findings show that serum Hcy levels did not change as a function of mood state in BD. The results also show that BMI is a determinant of Hcy levels. The significant influence of BMI on Hcy levels corroborates the results of a cohort study ${ }^{29}$; the results also show the importance of assessing this variable in future studies.

Median serum concentrations of folate and vitamin B12 were within normal range and did not differ between patients and controls. These levels did not change between the two mood states in the same patient. CRP levels were also within normal range when compared with controls.

The finding of lower ferritin values during manic episodes in comparison with euthymia and in comparison with controls has not been elucidated. Decreased ferritin has been associated with other psychiatric disorders, such as attention deficit-hyperactivity, schizophrenia and major depression. ${ }^{30-32}$ Studies have described direct proinflammatory effects of iron deficiency on macrophages and foam cells. ${ }^{33}$ Other methods can be used to estimate cellular iron stores in patients. The lower levels of ferritin in the manic state may be due to limited external supply of iron and/or vitamin C. Our patients did not report use of drugs or infections that could impair absorption of iron or be associated with blood loss.

The present study has specific limitations. The reduced sample size may have limited the statistical power necessary to show significant differences between the groups. The p-values obtained for CRP (0.056) do, however, suggest a trend toward higher levels of CRP in patients with BD in comparison with healthy controls. Furthermore, we chose to minimize the chance of obtaining false positives by using Bonferroni correction; this choice may increase the probability of producing false negatives. Another limitation of the study is the lack of information about other confounding variables, such as the number of previous episodes and lifestyle factors (eating habits and physical activity).

The influence of medication on Hcy levels is controversial. Studies have shown no significant differences in Hcy levels between patients administered lithium in comparison with those not administered lithium, neuroleptics or valproate. ${ }^{34}$

Given the substantial risk of cardiovascular disease, rigorous assessment of cardiovascular risk is warranted

Table 1 Biochemical parameters (mean \pm standard deviation)

\begin{tabular}{|c|c|c|c|c|c|}
\hline & \multicolumn{2}{|c|}{ Bipolar patients } & \multirow[b]{2}{*}{ Controls } & \multirow[b]{2}{*}{$p$-value } & \multirow[b]{2}{*}{ Adjusted p-value* } \\
\hline & Mania & Euthymia & & & \\
\hline Homocysteine ( $\mu \mathrm{mol} / \mathrm{L})$ & $7.74 \pm 0.99$ & $7.66 \pm 0.87$ & $4.17 \pm 0.63$ & $0.003^{\dagger}$ & 0.101 \\
\hline Vitamin B12 $(\rho \mathrm{g} / \mathrm{ml})$ & $425.04 \pm 33.93$ & $433.56 \pm 46.46$ & $345.40 \pm 19.12$ & 0.124 & 0.996 \\
\hline Folate $(\eta \mathrm{g} / \mathrm{ml})$ & $10.81 \pm 1.11$ & $9.50 \pm 0.60$ & $10.36 \pm 0.95$ & 0.334 & 0.622 \\
\hline Ferritin ( $\mathrm{gg} / \mathrm{ml})$ & $126.69 \pm 27.39$ & $172.74 \pm 32.27$ & $171.94 \pm 28.79$ & $0.044^{\dagger}$ & $0.016^{\dagger}$ \\
\hline C-reactive protein (mg/L) & $5.17 \pm 1.39$ & $4.69 \pm 1.43$ & $3.80 \pm 0.96$ & 0.701 & 0.056 \\
\hline Creatine kinase $(\mathrm{U} / \mathrm{L})$ & $175.84 \pm 45.98$ & $79.43 \pm 9.83$ & $92.67 \pm 17.19$ & $0.006^{\dagger}$ & 0.112 \\
\hline
\end{tabular}

\footnotetext{
* Adjusted by body mass index.

$\mathrm{p}<0.05$
} 
for patients with BD. Modifiable risk factors should be addressed when identified. Further research is needed to study the mechanisms underlying elevated risk of cardiovascular disease in this patient population. Delivery of medical and psychiatric care should be integrated for individuals with BD.

\section{Acknowledgments}

This study was supported by Fundo de Incentivo à Pesquisa - Hospital de Clínicas de Porto Alegre (FIPEHCPA).

\section{Disclosure}

Flavio Kapczinski has received grant/research support from Astra(Zeneca, Eli Lilly, Janssen-Cilag, Servier, Coordenaçäo de Aperfeiçoamento de Pessoal de Nível Superior (CAPES), Conselho Nacional de Desenvolvimento Científico e Tecnológico (CNPq), NARSAD, and the Stanley Medical Research Institute; has been a member of the speakers boards for Astra-Zeneca, Eli Lilly, Janssen and Servier; and has served as a consultant for Servier. The other authors report no conflicts of interest.

\section{References}

1 Osby U, Brandt L, Correia N, Ekbom A, Sparen P. Excess mortality in bipolar and unipolar disorder in Sweden. Arch Gen Psychiatry. 2001;58:844-50.

2 Fagiolini A, Frank E, Scott JA, Turkin S, Kupfer DJ. Metabolic syndrome in bipolar disorder: findings from the Bipolar Disorder Center for Pennsylvanians. Bipolar Disord. 2005;7:424-30.

3 Tsai SY, Lee CH, Kuo CJ, Chen CC. A retrospective analysis of risk and protective factors for natural death in bipolar disorder. J Clin Psychiatry. 2005;66:1586-91.

4 Fiedorowicz JG, He J, Merikangas KR. The association between mood and anxiety disorders with vascular diseases and risk factors in a nationally representative sample. J Psychosom Res. 2011;70:14554.

5 Veeranna V, Zalawadiya SK, Niraj A, Pradhan J, Ference B, Burack $\mathrm{RC}$, et al. Homocysteine and reclassification of cardiovascular disease risk. J Am Coll Cardiol. 2011;58:1025-33.

6 Wald DS, Law M, Morris JK. Homocysteine and cardiovascular disease: evidence on causality from a meta-analysis. BMJ. 2002;325:1202

7 Rasouli ML, Nasir K, Blumenthal RS, Park R, Aziz DC, Budoff MJ. Plasma homocysteine predicts progression of atherosclerosis. Atherosclerosis. 2005;181:159-65.

8 Schroecksnadel K, Frick B, Winkler C, Leblhuber F, Wirleitner B, Fuchs D. Hyperhomocysteinemia and immune activation. Clin Chem Lab Med. 2003;41:1438-43.

9 Voutilainen S, Rissanen TH, Virtanen J, Lakka TA, Salonen JT. Low dietary folate intake is associated with an excess incidence of acute coronary events: The Kuopio Ischemic Heart Disease Risk Factor Study. Circulation. 2001;103:2674-80.

10 Durga J, Bots ML, Schouten EG, Kok FJ, Verhoef P. Low concentrations of folate, not hyperhomocysteinemia, are associated with carotid intima-media thickness. Atherosclerosis. 2005;179:285-92.

11 Onat A, Hergenc G, Kucukdurmaz Z, Can G, Ayhan E, Bulur S. Serum folate is associated with coronary heart disease independently of homocysteine in Turkish men. Clin Nutr. 2008;27:732-9.

12 Suh JR, Herbig AK, Stover PJ. New perspectives on folate catabolism. Annu Rev Nutr. 2001;21:255-82.

13 Rugulies R. Depression as a predictor for coronary heart disease. a review and meta-analysis. Am J Prev Med. 2002;23:51-61.
14 Ferketich AK, Schwartzbaum JA, Frid DJ, Moeschberger ML. Depression as an antecedent to heart disease among women and men in the NHANES I study. National Health and Nutrition Examination Survey. Arch Intern Med. 2000;160:1261-8.

15 Fiedorowicz JG, Solomon DA, Endicott J, Leon AC, Li C, Rice JP, Coryell WH. Manic/hypomanic symptom burden and cardiovascular mortality in bipolar disorder. Psychosom Med. 2009;71:598-606.

16 Henry BL, Minassian A, Paulus MP, Geyer MA, Perry W. Heart rate variability in bipolar mania and schizophrenia. J Psychiatr Res. 2010;44:168-76

17 Carvajal K, Moreno-Sanchez R. Heart metabolic disturbances in cardiovascular diseases. Arch Med Res. 2003;34:89-99.

18 Cleland SJ, Sattar N, Petrie JR, Forouhi NG, Elliott HL, Connell JM. Endothelial dysfunction as a possible link between $\mathrm{C}$-reactive protein levels and cardiovascular disease. Clin Sci (Lond). 2000;98:531-5.

19 Sugano Y, Anzai T, Yoshikawa T, Satoh T, Iwanaga S, Hayashi T, et al. Serum C-reactive protein elevation predicts poor clinical outcome in patients with distal type acute aortic dissection: association with the occurrence of oxygenation impairment. Int $\mathrm{J}$ Cardiol. 2005;102:39-45.

20 Huang TL, Lee CT, Liu YL. Serum brain-derived neurotrophic factor levels in patients with major depression: effects of antidepressants. J Psychiatr Res. 2008;42:521-5.

21 Lyoo IK, Sung YH, Dager SR, Friedman SD, Lee JY, Kim SJ, et al. Regional cerebral cortical thinning in bipolar disorder. Bipolar Disord. 2006;8:65-74.

22 Magera MJ, Lacey JM, Casetta B, Rinaldo P. Method for the determination of total homocysteine in plasma and urine by stable isotope dilution and electrospray tandem mass spectrometry. Clin Chem. 1999;45:1517-22.

23 De Hert M, Dekker JM, Wood D, Kahl KG, Holt RI, Moller HJ. Cardiovascular disease and diabetes in people with severe mental illness position statement from the European Psychiatric Association (EPA), supported by the European Association for the Study of Diabetes (EASD) and the European Society of Cardiology (ESC). Eur Psychiatry. 2009;24:412-24.

24 Ebbing M, Bønaa KH, Arnesen E, Ueland PM, Nordrehaug JE, Rasmussen K, et al. Combined analyses and extended follow-up of two randomized controlled homocysteine-lowering B-vitamin trials. J Intern Med. 2010;268:367-82.

25 Herrmann M, Taban-Shomal O, Hubner U, Bohm M, Herrmann W. A review of homocysteine and heart failure. Eur $J$ Heart Fail. 2006:8:571-6

26 Osher Y, Sela BA, Levine J, Belmaker RH. Elevated homocysteine levels in euthymic bipolar disorder patients showing functional deterioration. Bipolar Disord. 2004;6:82-6.

27 Dittmann S, Seemuller F, Schwarz MJ, Kleindienst N, Stampfer R, Zach J, et al. Association of cognitive deficits with elevated homocysteine levels in euthymic bipolar patients and its impact on psychosocial functioning: preliminary results. Bipolar Disord. 2007;9:63-70.

28 Dias VV, Brissos S, Cardoso C, Andreazza AC, Kapczinski F. Serum homocysteine levels and cognitive functioning in euthymic bipolar patients. J Affect Disord. 2009;113:285-90.

29 Jacques PF, Bostom AG, Wilson PW, Rich S, Rosenberg IH, Selhub J. Determinants of plasma total homocysteine concentration in the Framingham Offspring cohort. Am J Clin Nutr. 2001;73:613-21.

30 Kuloglu M, Atmaca M, Ustundag B, Canatan H, Gecici O, Tezcan E. Serum iron levels in schizophrenic patients with or without akathisia. Eur Neuropsychopharmacol. 2003;13:67-71.

31 Oner O, Alkar OY, Oner P. Relation of ferritin levels with symptom ratings and cognitive performance in children with attention deficithyperactivity disorder. Pediatr Int. 2008;50:40-4.

32 Baune BT, Neuhauser $\mathrm{H}$, Ellert U, Berger K. The role of the inflammatory markers ferritin, transferrin and fibrinogen in the relationship between major depression and cardiovascular disorders - The German Health Interview and Examination Survey. Acta Psychiatr Scand. 2010;121:135-42.

33 Fan Y, Wang J, Wei L, He B, Wang C, Wang B. Iron deficiency activates pro-inflammatory signaling in macrophages and foam cells via the p38 MAPK-NF-kappaB pathway. Int J Cardiol. 2011;152:49-55.

34 Osher $\mathrm{Y}$, Bersudsky $\mathrm{Y}$, Silver $\mathrm{H}$, Sela BA, Belmaker $\mathrm{RH}$. Neuropsychological correlates of homocysteine levels in euthymic bipolar patients. J Affect Disord. 2008;105:229-33. 